\title{
ChemComm
}

Cite this: Chem. Commun., 2014 50, 1369

Received 23rd October 2013, Accepted 26th November 2013

DOI: $10.1039 / c 3 c c 48130 a$

www.rsc.org/chemcomm

\section{Drug delivery without nanoparticle uptake: delivery by a kiss-and-run mechanism on the cell membrane $\dagger$}

\author{
Daniel Hofmann, $\ddagger^{\mathrm{ab}}$ Claudia Messerschmidt, $\ddagger^{\mathrm{a}}$ Markus B. Bannwarth, ${ }^{\text {ac }}$ \\ Katharina Landfester ${ }^{a}$ and Volker Mailänder ${ }^{\star a b}$
}

\begin{abstract}
Nearly all concepts of nanocarriers as drug delivery devices rely on intracellular uptake. Instead, we demonstrate an alternative concept for rapid and specific delivery of cargo by nanoparticles to TIP47+/ ADRP+ lipid droplets. The model can serve as a novel strategy for the non-invasive delivery of drugs by releasing hydrophobic cargo, in our case a model dye, through a kiss-and-run mechanism between nanoparticles and the cell membrane.
\end{abstract}

Great efforts have been made to understand the interactions between cells and nanoparticle drug delivery devices, ${ }^{1,2}$ with a focus on uptake of nanoparticles and their intracellular trafficking pathways. To bypass the complex endocytotic uptake ${ }^{3-7}$ and intracellular transport of nanospheres to subcellular compartments via endocytotic vesicles, a direct way of cargo delivery right on the cell membrane could avoid this complexity. In the concept presented here the cargo release mainly occurs on the cellular membrane, which depends on parameters like hydro- and lipophilicity of the nanoparticles, the cargo and the lipid bilayer. Hydrophobic molecules that are loaded onto polymeric nanoparticles are hardly or non-water soluble impeding the simple diffusion out of the polymeric matrix. ${ }^{8}$ This opens up the possibility for the release on hydrophobic surfaces or media due to their lipophilic properties. Here such a mechanism has been observed and is described as a short touch and detach ("kiss-and-run") mechanism (Scheme 1). We demonstrate that nanoparticles made of long-term biodegradable polymers like the widely used poly-L-lactide (PLLA) ${ }^{9}$ or even of non-biodegradable

\footnotetext{
${ }^{a}$ Max Planck Institute for Polymer Research, Ackermannweg 10, 55128 Mainz, Germany. E-mail: volker.mailaender@mpip-mainz.mpg.de

${ }^{b}$ III. Medical Clinic, Hematology, Oncology and Pneumology,

University Medical Center of the Johannes-Gutenberg University Mainz, Langenbeckstr. 1, 55101 Mainz, Germany

${ }^{c}$ Graduate School Materials Science in Mainz, Staudinger Weg 9, 55128 Mainz, Germany

$\dagger$ Electronic supplementary information (ESI) available. See DOI: 10.1039/ c3cc48130a

\$ Shared first authorship.
}
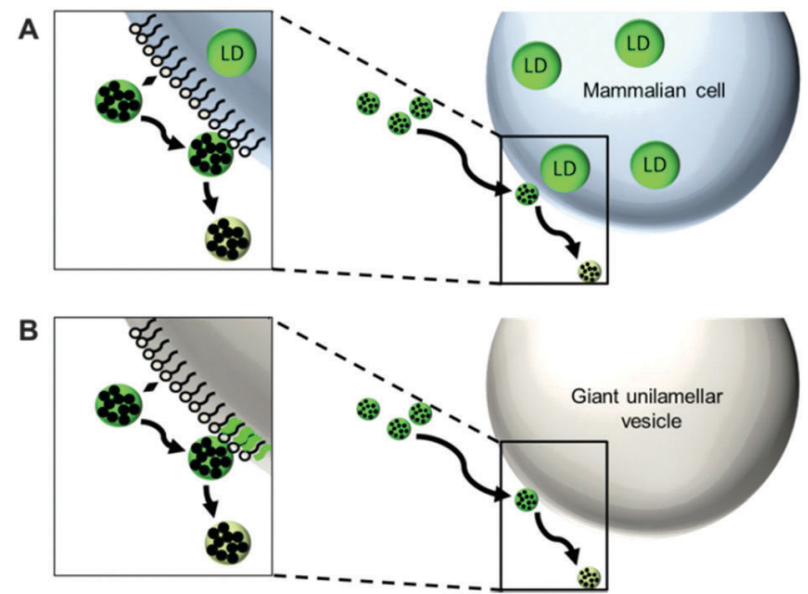

Scheme 1 Schematic illustration of the kiss-and-run mechanism. Nanoparticles briefly interact with the lipid bilayer of a cell (A) or a giant unilamellar vesicle (B), thereby releasing its dye that accumulates in lipid droplets (LD) or in the membrane, respectively.

polymers like polystyrene can transport a hydrophobic cargo in an aqueous environment while delivering the cargo within minutes and without entering the cell just by "touching" the phospholipid layer. We demonstrate that the cargo further accumulates inside lipid droplets, a subcellular compartment for lipid storage. In this context, we observed a convenient mechanism that comprises a rapid and membrane-specific delivery of hydrophobic substances with a specific release on the cell membrane.

For this reason, biodegradable PLLA nanoparticles were used to demonstrate the drug delivery abilities of such nanoparticles (for nanoparticle synthesis and characterization see ESI $\dagger$ ). To track the cargo release, the PLLA nanoparticles (PLLA-Fe-PMI) were labeled with iron oxide and a non-covalently bound fluorescent dye, perylene monoimide ( $N$-(2,6-diisopropylphenyl)perylene-3,4-dicarboximide, PMI). ${ }^{10}$ The dye was inserted to enable detection by confocal laser scanning microscopy (CLSM). The magnetite load was used to assist in detecting the nanoparticles by transmission 


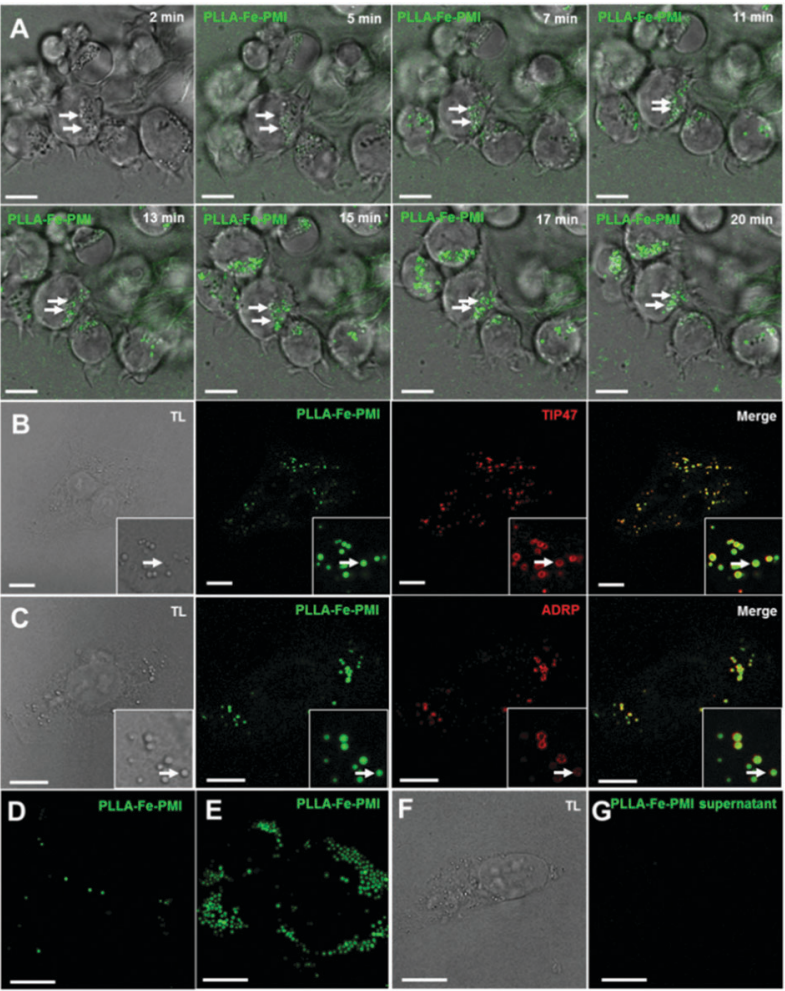

Fig. 1 Nanoparticle cargo is transported to lipid droplets. (A) Live cell imaging of Jurkat cells incubated with PLLA-Fe-PMI nanoparticles for $20 \mathrm{~min}$ in confocal microscopy (white arrows = lipid droplets under transmission light). (B) Tail-interacting protein 47 (TIP47) and (C) adipose differentiation-related protein (ADRP) surround the PMI-stained lipid droplets. Cells were incubated with PLLA-Fe-PMI for 15 min. (D) HeLa cells incubated with PLLA-Fe-PMI nanoparticles for 15 minutes. (E) HeLa cells were fed with $25 \mu \mathrm{M}$ oleic acid overnight followed by incubation of PLLA-Fe-PMI nanoparticles for $15 \mathrm{~min}$. ( $F$ and $G$ ) $2 \mathrm{~h}$ incubation of the PLLA-Fe-PMI nanoparticle supernatant (scale bar $=10 \mu \mathrm{m}$ ).

electron microscopy (TEM) and could be used for magnetic resonance imaging. ${ }^{11}$ Confocal imaging revealed a remarkably fast transport within minutes of the hydrophobic perylene monoimide into perfectly round shaped intracellular compartments with a high refractive index as demonstrated by differential interphase contrast (DIC) microscopy (Fig. 1A-C). This fluorescence appeared after the start of the incubation with the first signals seen as early as 10-30 min in HeLa cells (Fig. 1B and C). Jurkat cells (a non-adherent $\mathrm{T}$ cell line) were used as a totally different cell type to confirm these results. In live cell imaging experiments with Jurkat cells we observed a strong staining of these intracellular compartments, seen under transmission light after 5-20 min (Fig. 1A).

In contrast, and despite extensive TEM studies, even after 30 min of incubation HeLa cells do not contain nanoparticles inside the cells as shown in TEM experiments (Fig. S2B, ESI†). No fluorescent signal was detectable after the incubation of the nanoparticle supernatant with HeLa cells excluding a staining of the cells by free dye molecules (Fig. 1F and G). To investigate the localization of the fluorescent signal further, we used a correlative microscopic approach to generate an overlay micrograph of confocal and transmission electron microscopy results of a single cell. This revealed a localization of nanoparticles on the cell membrane for both methods. Most importantly, we were not able to detect nanoparticles in the brightly stained intracellular regions but detected extracellular fluorescent nanoparticles partially interacting with the cell membrane (Fig. S2, ESI $†$ ).

Taken together, and keeping the DIC microscopic appearance in mind, we suspected that the highly diffractive intracellular compartments could be lipid droplets. ${ }^{12}$ By feeding cells with BSA-coupled oleic acid the number of lipid droplets inside HeLa cells can be increased. ${ }^{13}$ After nanoparticle incubation, the fluorescence from the dye PMI was found inside these highly diffractive organelles that had increased in number and size due to the treatment (Fig. 1E). In order to confirm this further, subcellular localization studies with immunofluorescence staining of two specific membrane markers of lipid droplets, tailinteracting protein 47 (TIP47) and adipose differentiation-related protein (ADRP) around the PMI signal were investigated. ${ }^{13,14}$ This demonstrated a high degree of overlap of fluorescent PMI and the lipid droplet specific markers (Fig. 1B and C). Transmission light analysis identified PMI/TIP47/ADRP colocalization with organelles of a high diffractive index representing lipid droplets in comparison to the rest of the cell (Fig. 1A, B and E). From these observations we could conclude that the hydrophobic molecule is released into the phospholipid membrane of the cell and is then localized into even more hydrophobic intracellular compartments - namely lipid droplets as mentioned earlier.

To evaluate the first step in a cell free system, PMI release in different biological and hydrophobic media was analyzed. We observed a controlled release of PMI out of PLLA-Fe-PMI nanoparticles only in glyceryl trioleate, but not in 1\% DMSO solution and cell culture media with fetal calf serum (Fig. 2A). This demonstrates that the nanoparticles preferably release their hydrophobic cargo molecules inside lipophilic environments but lack release in hydrophilic environments. Next, we performed membrane release experiments with giant unilamellar vesicles made of 1,2-dioleoyl-sn-glycero-3-phosphocholine (DOPC) to simulate a hydrophobic cell membrane that a nanoparticle can interact with. These experiments were performed in pure water with PLLA-Fe-PMI (PLLA-Fe-PMI, Fig. 2B), covalently dye-labeled

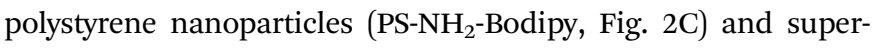
paramagnetic iron oxide nanoparticles (SPIOPSN-Bodipy; Fig. S3, ESI†). PLLA-Fe-PMI nanoparticles interact for some $100 \mathrm{~ms}$ with the DOPC membrane leaving the membrane after this short touching event (Fig. 2C, white arrows). After 15-20 min, the DOPC membrane was fluorescently labeled by PMI. We referred to this event as a brief "kiss", leaving behind some stains on the touched object. After its "kiss", the particle detaches and "runs" away.

In the control experiments with PLLA-Fe-PMI supernatants and polystyrene nanoparticles containing covalently bound dyes, no staining of the DOPC GUVs was observed (Fig. 2B and D). With PS- $\mathrm{NH}_{2}$-Bodipy, we investigated a polystyrene nanoparticle with a covalently bound dye that strongly interacts with the giant vesicle by dashing around on the surface of the vesicle without releasing the covalently bound dye Bodipy (Fig. 2D, white arrows). In Fig. S3 (ESI $\dagger$ ), it can be seen that the superparamagnetic nanoparticle 


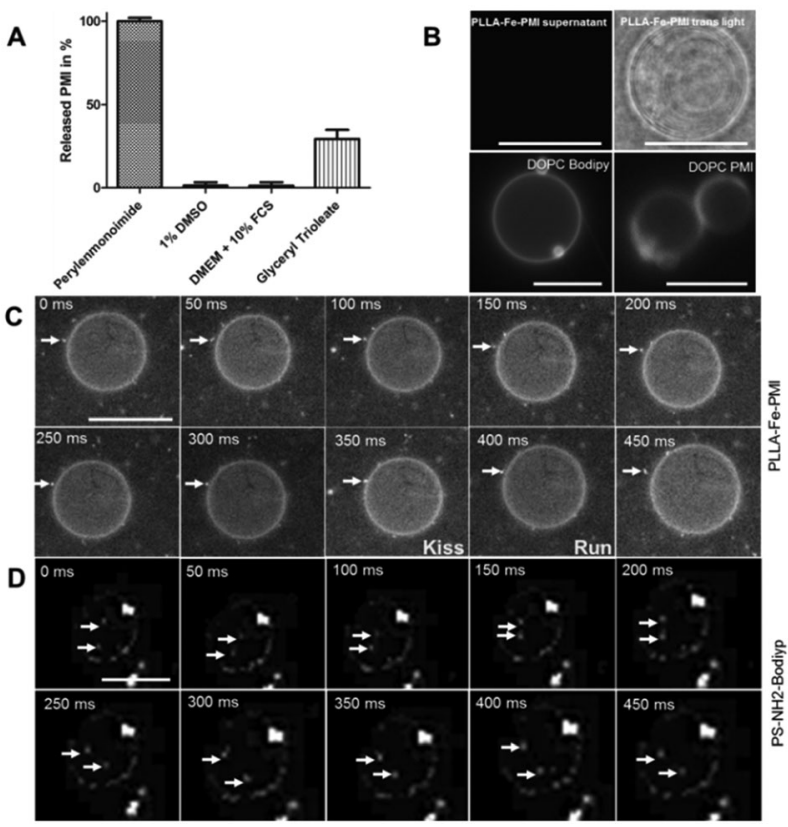

Fig. 2 Release studies of PMI in hydrophobic/hydrophilic media and on giant unilamellar vesicles. (A) PMI release from PLLA-Fe-PMI nanoparticles was tested in different media. (B) Supernatant of PLLA-Fe-PMI nanoparticles after $2 \mathrm{~h}$ of incubation with DOPC-GUVs. Staining of DOPC-GUVs by free Bodipy and PMI. (C) 15 min incubation of PLLA-Fe-PMI-magnetite nanoparticles with DOPC-GUVs. White arrows indicate kiss-and-run movement of nanoparticles. (D) 15 min incubation of polystyrene nanoparticles with covalently bound Bodipy, floating onto DOPC-GUVs. Micrographs were taken after $30 \mathrm{~min}$ of nanoparticle-vesicle interaction. The frame rate was $20 \mathrm{~s}^{-1}$ (scale bar $=10 \mu \mathrm{m}$ ).

is not permanently interacting with the surface of the giant vesicle. Nevertheless, also a kiss-and-run-like mechanism without the release of the dye into the lipid membrane was observed (Fig. S3, ESI $\dagger$ ).

Therefore, we conclude that our PMI-containing nanoparticles briefly interact with the phospholipid bilayer of cells and vesicles releasing the hydrophobic cargo. In the case of cells, PMI diffuses through the membrane and by membrane turnover accumulates in the even more hydrophobic surrounding of lipid droplets, while in the case of GUVs, only the membrane is stained.

In summary, we observed (1) a temporary interaction of nanoparticles with hydrophobic membrane surfaces, (2) a fast release of hydrophobic molecules from the particle when encountering a membrane and (3) the terminal fate of the cargo localizing in lipid droplets. These parameters describe a novel drug delivery mechanism ("kiss-and-run") that may change the paradigm of drug delivery. Contrary to most concepts we show that no nanoparticle uptake is necessary, but only a short contact (about $100 \mathrm{~ms}$ ) with the cell membrane to deliver a hydrophobic molecule. Cargo release was induced due to the interaction of the nanoparticle with hydrophobic surroundings and lipophilic surfaces and detectable after several minutes. Our data suggest a mechanism that results in the specific subcellular delivery of hydrophobic molecules into lipid droplets by the induced release on cell membranes and reveals that nanomaterials exchange components of their own when encountering membraneous systems. The unspecificity and stochastic nature of the kiss-and-run mechanism calls for the design of more complex nanoparticles (e.g. targeted core-shell structures) for applications in vivo as the cargo should be released on the desired cell type and not on the first cell membrane, e.g., capillary endothelial cells, encountered by the nanoparticle. A first possible application could be the subcellular targeting of lipid droplets to influence the lipid metabolism of dyslipidemic patients (e.g. niacin-conjugate delivery). ${ }^{15}$

This work was supported by the Deutsche Forschungsgesellschaft (SPP1313; LA1013/13-1; MA 3271/3-1) and the Max-Planck Society. M.B. Bannwarth is a recipient of a fellowship funded through the Excellence Initiative (DFG/GSC 266).

\section{Notes and references}

1 A. Lesniak, A. Salvati, M. J. Santos-Martinez, M. W. Radomski, K. A. Dawson and C. Aberg, J. Am. Chem. Soc., 2013, 135, 1438-1444.

2 A. H. Faraji and P. Wipf, Bioorg. Med. Chem., 2009, 17, 2950-2962.

3 J. Lu, M. Liong, J. I. Zink and F. Tamanoi, Small, 2007, 3, 1341-1346.

4 A. M. Sauer, A. Schlossbauer, N. Ruthardt, V. Cauda, T. Bein and C. Brauchle, Nano Lett., 2010, 10, 3684-3691.

5 V. Cauda, H. Engelke, A. Sauer, D. Arcizet, C. Brauchle, J. Radler and T. Bein, Nano Lett., 2010, 10, 2484-2492.

6 K. G. de Bruin, C. Fella, M. Ogris, E. Wagner, N. Ruthardt and C. Brauchle, J. Controlled Release, 2008, 130, 175-182.

7 D. Hofmann and V. Mailander, Nanomedicine, 2013, 8, 321-323.

8 M. A. Augustin, L. Sanguansri and T. Lockett, Ann. N. Y. Acad. Sci., 2013, 1290, 107-112.

9 J. Panyam and V. Labhasetwar, Adv. Drug Delivery Rev., 2003, 55, 329-347.

10 V. Holzapfel, A. Musyanovych, K. Landfester, M. R. Lorenz and V. Mailander, Macromol. Chem. Phys., 2005, 206, 2440-2449.

11 G. Schmidtke-Schrezenmeier, M. Urban, A. Musyanovych, V. Mailander, M. Rojewski, N. Fekete, C. Menard, E. Deak, K. Tarte, V. Rasche, K. Landfester and H. Schrezenmeier, Cytotherapy, 2011, 13, 962-975.

12 R. V. Farese Jr. and T. C. Walther, Cell, 2009, 139, 855-860.

13 A. V. Bulankina, A. Deggerich, D. Wenzel, K. Mutenda, J. G. Wittmann, M. G. Rudolph, K. N. Burger and S. Honing, J. Cell Biol., 2009, 185, 641-655.

14 C. Londos, D. L. Brasaemle, C. J. Schultz, J. P. Segrest and A. R. Kimmel, Semin. Cell Dev. Biol., 1999, 10, 51-58.

15 A. Sharma, A. Khatchadourian, K. Khanna, R. Sharma, A. Kakkar and D. Maysinger, Biomaterials, 2011, 32, 1419-1429. 